\title{
Multipath Separation and Parameter Estimation by Single RIS Antenna in Fading Channel
}

\author{
$1^{\text {nd }}$ Yangming Lou \\ PLA Strategic Support Force \\ Information Engineering University \\ Zhengzhou, China \\ louyangming1991@outlook.com
}

\author{
$4^{\text {th }}$ Zhou Zhong \\ PLA Strategic Support Force \\ Information Engineering University \\ Zhengzhou, China \\ zhongzhou1016@vip.qq.com
}

\author{
$2^{\text {st }}$ Liang Jin \\ PLA Strategic Support Force \\ Information Engineering University \\ Zhengzhou, China \\ liangjin@263.net
}

\author{
$3^{\text {rd }}$ Xiaoming Xu \\ PLA Strategic Support Force \\ Information Engineering University \\ Zhengzhou, China \\ ee_xiaomingxu@sina.com
}

\begin{abstract}
Channel identification and measurement accuracy will greatly affect wireless communication performance. The broadcasting of electromagnetic waves will produce multipath, and its superposition will lead to fading. If the multipath can be separated and the phase of each path can be adjusted separately, better signal transmission and reception strategies can be designed to weaken or eliminate fading and improve signal transmission performance. In this paper, we first analyze the multipath structure of the wireless channel, then utilize the dynamic isomeric characteristics of the reconfigurable intelligent surface (RIS) antenna, also called dynamic metamaterial antenna (DMA), to form differentiated patterns, and rapidly samples within a period of single pilot symbol, so that a group of multipath signals are projected on multiple patterns and paths can be separated and measured. We simulated and evaluated the normalized mean square error of the parameter estimates, including angle of arrival and path gain of channels under different conditions. The results demonstrate that even single 1-bit coding RIS antenna can achieve well multipath separation and parameter estimation effects.
\end{abstract}

\section{INTRODUCTION}

Without the shackles of cables, wireless communication had obtained more widespread application in so many scenarios. In recent years, with the development and commercialization of 5G/B5G and other technologies, the main technology parameters of wireless communication such as transmission rate and time delay have gradually approached those of wired communication, resulting in new applications such as the Internet of Things, vehicle networking, and telemedicine. These innovations provide convenience and civilized lifestyle. Because electromagnetic waves are used to broadcast information, wireless communication will inevitably be greatly affected by the environment. Various terrain will not only cause the attenuation of energy, but also cause the generation of signal multipath, resulting in fading, and signal distortion at receiver, reducing the communication performance. To deal with these problems, channel estimation become an indispensable link in wireless communication systems.
The narrowband wireless communication system can be simply described as

$$
\boldsymbol{y}=\boldsymbol{H} \boldsymbol{x}+\boldsymbol{n}
$$

where $\boldsymbol{n}$ is noise, $\boldsymbol{y}, \boldsymbol{x}$ denote the reception and transmission of wireless signals, respectively. $\boldsymbol{H}$ represents channel, which is a matrix in a multi-antenna system, where each element represents a channel between a pair of transmit and receive antennas. In the conventional channel estimation method, $\boldsymbol{x}$ is a known reference signal, which is used to measure the wireless channel during transmission. The receiver estimates channel parameters according to the received signal $\boldsymbol{y}$, compensates for the remaining received signals. The receiver can also feed back the result to transmitter, so that it can also compensate the signal before transmission. Classical channel estimation methods such as LS, MMSE, LMMSE, etc., have been widely used in engineering practice. The more precise and accurate the channel estimation, the more communication performance can be improved.

As shown in the Figure 1, the electromagnetic wave will produce multipath in the propagation process, and then enter the receiver after experiencing different multipath channels. In addition to the attenuation of energy, the multipath effect will also cause non-in-phase or even anti-phase superposition when the signal reaches receiving antenna, resulting in fading and deterioration of the signal-to-noise ratio (SNR). However, if multipath separation is possible, adjusting the phase of the arrival signal of each path separately can eliminate fading, thereby designing a better signal transmission and reception strategy, and improving performance of communication. In existing research, The measurement of multipath channels mainly relies on the joint angle and delay estimation method (JADE), which utilize the delay difference of paths to distinguish the multipath [1]. This method relies on the array antenna technology and improves the measurement accuracy by increasing the number of antennas, which has a relatively large engineering implementation complexity. In addition, under the 
condition of narrowband, the delay difference between paths is small, which makes it difficult to jointly estimate channel amplitude, phase, and angle information. Therefore, the method of separation and parameter estimation of multipath is seldom deeply studied.

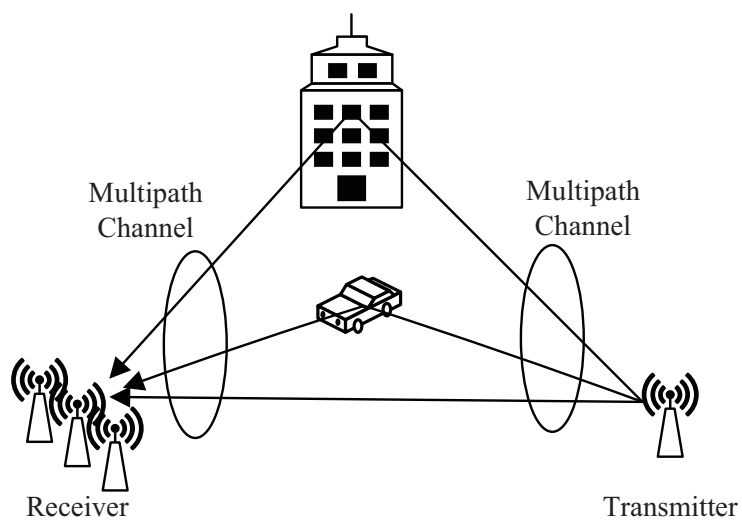

Fig. 1: In the process of electromagnetic wave propagation, the scattering, reflection, and refraction of electromagnetic waves by terrain and objects will cause the generation of multipath.

In recent years, the emergence of reconfigurable intelligent surface (RIS) has attracted widespread attention in the field of wireless communications [2]. RIS is a new type of electromagnetic device composed of a large number of metamaterial elements. Each element can adjust parameters such as the amplitude, phase, and polarization of electromagnetic waves. RIS has become a popular candidate technology in B5G/6G by virtue of its excellent electromagnetic wave control ability, small size and flexible deployment capability [3]. RIS has a variety of applications, and using it as a reflective surface is the main research direction at present. In scenario, RIS is usually deployed between transmitter and receiver as a passive device to shape the radio propagation environment, thereby expanding the signal coverage and increasing the system capacity [4]-[9]. Another more "active" way is to directly merge RIS and radio frequency devices to form a RIS antenna, which is also called a dynamic metamaterial antenna (DMA) in [10]-[12]. Antenna is the sensor of electromagnetic waves. RIS antenna utilizes metamaterials to control electromagnetic waves in analog domain, which enhances the signal processing capabilities of radio frequency front-end, and enables the receiver to observe and perceive the wireless environment more elaborately. The emergence of this new type of electromagnetic device provides fresh ideas for multipath channel estimation.

When the antenna receives electromagnetic signals, it has a differentiated reception effect for incoming waves from different directions, and this difference is reflected in the antenna pattern. At present, each element in the array has same structure, which makes them have the same pattern. This type of antenna array is called a isomorphic array, which relies on the difference in position of elements to sense electromagnetic waves. RIS, on the other hand, is a space-time isomorphic array, composed of a large number of metamaterial elements, each of which can rapidly and elaborately adjust its own pattern [13]. In space-time isomeric array, the pattern of elements not only differs in spatial dimension, but can also change rapidly in time dimension, enabling elements to achieve differential sampling at the same location to improve electromagnetic wave perception ability. In this paper, we will utilize the agility of isomeric array to realize the observation and separation of multipath channels. As shown in Figure 2, the multipath signal arrives at RIS from different directions, and then flows into the RF-chain after being affected by the pattern. This process can be regarded as the projection of paths on the pattern function of RIS antenna. Metamaterial elements rapidly change their states, forms differentiated patterns, receive the same set of multipath signals, which is equivalent to multi-directional projection, so that the paths can be separated and measured from the sampling results.

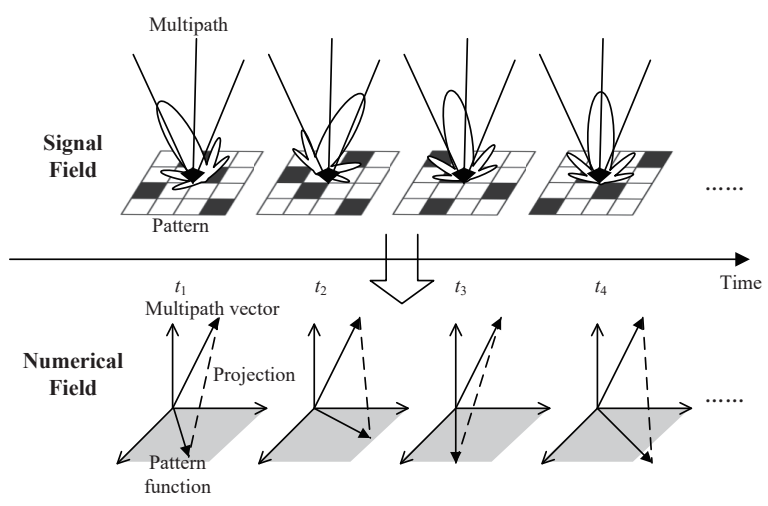

Fig. 2: The different metamaterial elements on RIS antenna utilize state agility to form differentiated directional patterns, and change multiple times within a single symbol period to receive the same group of multipath signals, realizing spacetime two-dimensional joint oversampling. This process can be regarded as the projection of multipath signal on the pattern function.

This paper focuses on the problem of multipath separation and channel parameter estimation in wireless communication systems under fading conditions. Different from the existing antenna array system, it relies on the dynamic isomeric capability of a single RIS antenna, it can sense the wireless environment, separate the multipath channels, and estimate the channel parameters including amplitude, phase and angle of arrival (AoA).

The contributions of the paper are summarized as follows:

- Establish an angle-dependent multipath channel model based on RIS antenna. Utilize the pattern agility of the RIS antenna to achieve multiple sampling within a single pilot symbol period, obtain equivalent multidimensional received signals, and achieve high-precision multipath channel observation. For the sake of simplicity and without loss of generality, the metamaterial elements on the RIS antenna are arranged in a one-dimensional 
uniform linear array (ULA) [10], and the result can be simply extended to a two-dimensional planar array.

- A joint estimation method of multipath channel parameters based on RIS antenna is proposed. Utilizing the spatial sparsity of the multipath channel, the method based on the atomic norm minimization theory for joint estimation of the amplitude, phase and angle of the multipath channel is realized. The proposed method can also be realized by 1-bit coding RIS, and compared with the existing multipath estimation methods, multipath channel estimation is realized without relying on the multipath delay.

The rest of the paper is organized as follows: Section II introduces the system model, followed by multipath channel estimation algorithm in Section III. The numerical results and performance evaluation is offered in Section IV. Section V draws the conclusions and discusses the potential directions for future investigation.

Notations: A bold lowercase $\boldsymbol{a}$ denotes the column vector, a bold capital letter $\boldsymbol{A}$ denotes the matrix, $(\cdot)^{T},(\cdot)^{H}$ and $(\cdot)^{*}$ denote the transpose, Hermitian transpose, conjugate, respectively, the matrix inverse and Moore-Penrose pseudoinverse are denoted by $(\cdot)^{-1},(\cdot)^{\dagger}$, respectively, $\|\cdot\|_{2}$ is $l_{2}$ norm, $\operatorname{diag}(\boldsymbol{a})$ denotes a square diagonal matrix with entries of a on its diagonal, $\operatorname{tr}(\boldsymbol{A})$ denotes the trace, the sum value of the diagonal elements of $\boldsymbol{A}, \mathbb{C}$ denotes complex field.

\section{System Model}

In Figure 3, consider the communication between two single-antenna transceivers, denoted as Alice and Bob respectively. Alice is equipped with a RIS antenna containing $N$ elements for elaborate measurement of multipath channels. During the signal propagation, due to the influence of terrain on the electromagnetic wave, the signal transmit by Bob will go through multiple paths to reach the RIS antenna.

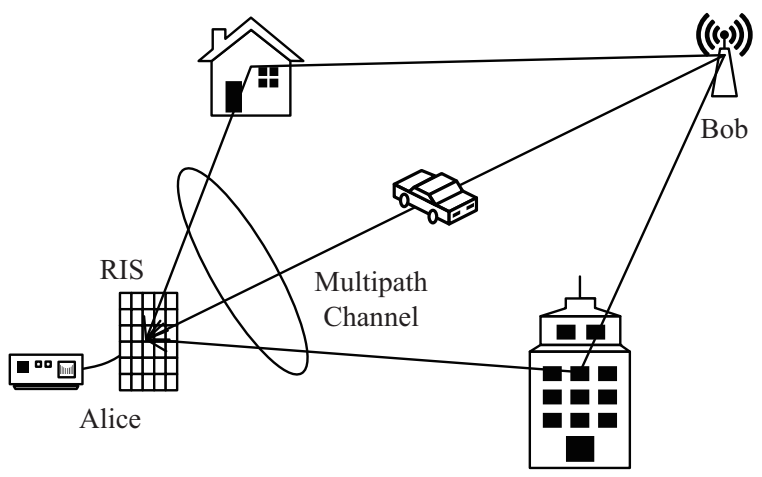

Fig. 3: Communication between Alice and Bob, where Alice is equipped RIS antenna while Bob is equipped conventional single antenna.

We use the AoA and complex gain of each path to describe the multipath channel. Wireless channel is modeled as

$$
h_{B A}=\boldsymbol{\phi}^{T}(\boldsymbol{\theta}) \boldsymbol{h}_{B R}(\boldsymbol{\theta})
$$

where $\boldsymbol{h}_{B R}(\boldsymbol{\theta}) \in \mathbb{C}^{L \times 1}$ is the channel between Bob and Alice, the number of paths is $L$, and $\boldsymbol{\theta}=\left[\theta_{1}, \ldots, \theta_{L}\right]^{T}$ represents the multipath AoA at Alice, $\boldsymbol{h}_{B R}(\boldsymbol{\theta}) \triangleq\left[h\left(\theta_{1}\right), \ldots, h\left(\theta_{L}\right)\right]^{T}$. $\boldsymbol{\phi}(\boldsymbol{\theta}) \in \mathbb{C}^{L \times 1}$ is the pattern response of RIS antenna at $\boldsymbol{\theta}$, $\boldsymbol{\phi}(\boldsymbol{\theta}) \triangleq\left[\phi\left(\theta_{1}\right), \ldots, \phi\left(\theta_{L}\right)\right]^{T}$. The ultimate goal of multipath channel estimation is to obtain the amplitude, phase and angle information of each path, namely $\boldsymbol{h}_{B R}(\boldsymbol{\theta})$ and $\boldsymbol{\theta}$.

To simplify the analysis, as shown in Figure 4, assume that the RIS antenna is a ULA formed by $N$ columns of metamaterial elements. The pattern response is

$$
\boldsymbol{\phi}^{T}(\boldsymbol{\theta})=\boldsymbol{\omega}^{T} \boldsymbol{A}(\boldsymbol{\theta})
$$

where $\boldsymbol{\omega} \triangleq\left[\omega_{1}, \ldots, \omega_{N}\right]^{T}, \omega_{n} \in \mathbb{C}$ represents the amplitude and phase of the incident electromagnetic wave changed by metamaterial elements, which is determined by the configuration parameters of RIS. $\boldsymbol{A}(\boldsymbol{\theta})$ is the manifold matrix, $\boldsymbol{A}(\boldsymbol{\theta}) \triangleq\left[\boldsymbol{a}\left(\theta_{1}\right), \ldots, \boldsymbol{a}\left(\theta_{L}\right)\right]$, where $\boldsymbol{a}(\theta)=$ $\left[1, \ldots, e^{j 2 \pi \frac{d}{\lambda} \sin \theta(N-1)}\right]^{T}$. The pattern of RIS antenna can be customized by adjusting the value of $\boldsymbol{\omega}$. It should be noted that RIS has different electromagnetic wave adjustment capabilities according to control methods [14]. Varactor diode based RIS has a strong ability to continuously adjust amplitude, phase, and polarization. However, its speed of adjustment is relatively slow, taking about hundreds of nanoseconds. In contrast, the states of PIN diode based RIS can only be switched between several discrete adjustment parameters but has a very fast state change speed, which can realize state switching within tens of nanoseconds, and has lower complexity to implement. Both types of RIS can meet the requirements of the method presented in this paper.

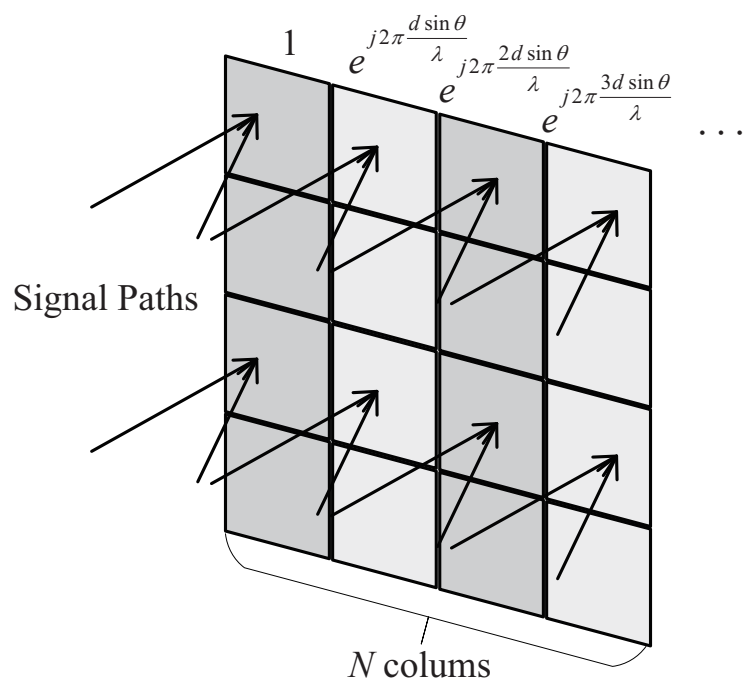

Fig. 4: RIS antenna is a ULA formed by $N$ columns of metamaterial elements.

The RIS antenna can change the pattern response $\phi(\theta)$ multiple times in a short time, even within a single symbol period, to achieve fast sampling. Assuming that the channel is unchanged during the estimation process, when sampling 
a single symbol for $M$ times, the channel represented by (2) can be rewritten as follows

$$
\boldsymbol{h}_{B A}=\boldsymbol{\Omega}^{T} \boldsymbol{A}(\boldsymbol{\theta}) \boldsymbol{h}_{B R}(\boldsymbol{\theta})
$$

$\boldsymbol{\Omega} \triangleq\left[\boldsymbol{\omega}_{1}, \ldots, \boldsymbol{\omega}_{M}\right]$, in $\boldsymbol{\Omega}, \boldsymbol{\omega}_{m}$ represents the RIS antenna state parameters used for sampling at $t_{m}$. It can be seen from (4) that there is a linear transformation relationship between $\boldsymbol{h}_{B R}(\boldsymbol{\theta})$ and $\boldsymbol{h}_{B A}$, that is, $\boldsymbol{h}_{B A}$ is the projection of $\boldsymbol{h}_{B R}(\boldsymbol{\theta})$. In (2), the projection $h_{B A}$ has lower dimension than $\boldsymbol{h}_{B R}(\boldsymbol{\theta})$, resulting in information loss. Therefore, it is impossible to restore $\boldsymbol{h}_{B R}(\boldsymbol{\theta})$ from $h_{B A}$. But in (4), the multi-dimensional output $\boldsymbol{h}_{B A}$ can be obtained through the rapid change of RIS antenna within the duration of a single received symbol. When the observation information is sufficient, there is no loss in the projection. Therefore, $\boldsymbol{h}_{B R}(\boldsymbol{\theta})$ can be recovered from it.

\section{Channel Estimation}

In this section, we will show up our channel estimation scheme. The entire scheme is divided into two stages: 1) Pilot signal processing; 2) Multipath channel parameter estimation based on atomic norm. We assume that the wireless channel is quasi-static block fading, so the channel remains unchanged during the entire channel estimation and subsequent data transmission. The data frame structure is shown in Figure 5.

\begin{tabular}{|l|l|l|l|}
\hline \multicolumn{2}{|c|}{ Pilot Signal } & \multicolumn{2}{c|}{ Data Signal } \\
\hline \multicolumn{3}{|l|}{} \\
\hline Symbol 1 & & $\ldots$ & Symbol $K$ \\
\hline \multicolumn{3}{|l|}{} & \\
\hline$\omega_{1}$ & $\ldots$ & $\omega_{M}$ & \\
\hline
\end{tabular}

Fig. 5: The data frame is composed of $K$ pilot symbols, and transmission data. In each pilot symbol period, the pattern of RIS antenna change $M$ times.

The first half of the data frame is composed of $K$ pilot symbols, and the second half is the transmission data. In each pilot symbol period, the pattern of RIS antenna change $M$ times, so Alice can get $K \times M$ samples, which can be used for channel estimation.

\section{A. Pilot signal processing}

In the $K$ th pilot symbol duration, the RIS antenna quickly changes the metamaterial element state $M$ times, then

$$
y_{k m}=\boldsymbol{\omega}^{T} \boldsymbol{A}(\boldsymbol{\theta}) \boldsymbol{h}_{B R}(\boldsymbol{\theta}) s_{k}+n_{k m}
$$

where $y_{k m}$ is the received signal during the $m$ th rapid change of the RIS antenna when the $k$ th pilot symbol is sent. $k m=$ $\sum_{i=1}^{N} n_{k m}^{(i)}$ is noise, which is the sum of received noise $n_{k m}^{(i)}$ on each metamaterial element. $n_{k m}^{(i)}$ are i.i.d, $n_{k m}^{(i)} \sim \mathcal{C N}\left(0, \sigma_{n}^{2}\right)$. Arrange $M$ received signals of Alice into a column vector $\boldsymbol{y}_{k}$,

$$
\begin{aligned}
\boldsymbol{y}_{k} & =\boldsymbol{h}_{B A} s_{k}+\boldsymbol{n}_{k} \\
& =\boldsymbol{\Omega}^{T} \boldsymbol{A}(\boldsymbol{\theta}) \boldsymbol{h}_{B R}(\boldsymbol{\theta}) s_{k}+\boldsymbol{n}_{k}
\end{aligned}
$$

$s_{k} \in \mathbb{C}$ is the pilot signal sent by Bob, $\boldsymbol{n}_{k} \triangleq\left[n_{k 1}, \ldots, n_{k M}\right]^{T}$. $s_{k}$ is a known signal, so $\boldsymbol{h}_{B A}$ can be obtained by using channel estimation algorithms such as LS, MMSE, LMMSE, etc. Better estimation algorithms can effectively improve the performance of multipath channel separation and parameter estimation, but will not affect our whole scheme. Without loss of generality, in this paper we use LS method, and the channel estimation result can be obtained as followed

$$
\tilde{\boldsymbol{h}}_{B A}=\frac{\boldsymbol{Y} \boldsymbol{s}^{*}}{\|\boldsymbol{s}\|_{2}}
$$

where $\boldsymbol{Y}_{M \times K} \triangleq\left[\boldsymbol{y}_{1}, \ldots, \boldsymbol{y}_{K}\right], \boldsymbol{s} \triangleq\left[s_{1}, \ldots, s_{K}\right]^{T}$ represents the pilot sequence. Essentially, the LS algorithm averages the samples of channels over time, thereby reducing the influence of noise.

Through (7), we have obtained the spatial multi-dimensional samples that removes the influence of noise as much as possible, and hope to recover $\boldsymbol{h}_{B R}$ from $\tilde{\boldsymbol{h}}_{B A} . \boldsymbol{F}(\boldsymbol{\theta}) \triangleq \boldsymbol{\Omega}^{T} \boldsymbol{A}(\boldsymbol{\theta})$, then

$$
\tilde{\boldsymbol{h}}_{B A}=\boldsymbol{F}(\boldsymbol{\theta}) \boldsymbol{h}_{B R}(\boldsymbol{\theta})+\tilde{\boldsymbol{n}}
$$

$\tilde{\boldsymbol{n}}$ represents residual noise. We can obtain the estimation of $\boldsymbol{h}_{B R}$ by solving following minimization problem,

$$
\min _{\boldsymbol{h}_{B R}(\boldsymbol{\theta})}\left\|\boldsymbol{F}(\boldsymbol{\theta}) \boldsymbol{h}_{B R}(\boldsymbol{\theta})-\tilde{\boldsymbol{h}}_{B A}\right\|_{2}
$$

For RIS antennas, paths may come from any direction facing the array, so $\theta_{l} \in[-\pi / 2, \pi / 2]$. Sample $[-\pi / 2, \pi / 2]$ at the interval of $\Delta \theta=\pi / P$ to get the sequence $\tilde{\boldsymbol{\theta}} \triangleq\left[\tilde{\theta}_{1}, \ldots, \tilde{\theta}_{P}\right]$, recorded as assumed directions. When the value of $P$ is large enough, the real direction $\theta_{l}$ will be very close to the assumed one $\tilde{\theta}_{p}$, which can be estimated by $\boldsymbol{h}_{B R}(\tilde{\boldsymbol{\theta}})$ instead of $\boldsymbol{h}_{B R}(\boldsymbol{\theta})$. Then the problem becomes

$$
\min _{\boldsymbol{h}_{B R}(\tilde{\boldsymbol{\theta}})}\left\|\boldsymbol{F}(\tilde{\boldsymbol{\theta}}) \boldsymbol{h}_{B R}(\tilde{\boldsymbol{\theta}})-\tilde{\boldsymbol{h}}_{B A}\right\|_{2}
$$

Obviously, in order to obtain a sufficiently accurate estimation result, the value of $P$ needs to be large enough, so that in $\boldsymbol{F}(\tilde{\boldsymbol{\theta}})$, the number of columns inevitably exceed the number of rows. It will make (8) become an underdetermined equation solving problem, which is really hard to solve in the usual sense. However, in most cases, the DoA of paths is much less than the number of assumed directions, that is, paths are sparse in the entire space. When some conditions are satisfied, the compressed sensing (CS) theory can be used to solve the problem [15]-[18].

\section{B. Multipath Channel Parameter Estimation based on Atomic Norm}

Conventional CS algorithms, such as orthogonal matching pursuit (OMP), were originally used in image processing. These methods are mainly for discrete signal processing. When applied to continuous signal, it is necessary to discretize the entire range as in (10), which is called grid method. But for multipath, although it is sparse in space, it may come from any angle within a certain range, so the value is continuous. When grid division is not fine enough, there will be a mismatch between the grid and the actual angle, which will affect the accuracy of result. These problems have been solved by gridless methods such as atomic norm minimization (ANM), 
which have been studies in the continuous signal processing such as DoA estimation, and the angle of multipath channels can be estimated based on this theory.

To simplify the calculation, it can be obtained from (8) that

$$
\begin{aligned}
\hat{\boldsymbol{h}}_{B A} & =\left(\boldsymbol{\Omega}^{T}\right)^{\dagger} \tilde{\boldsymbol{h}}_{B A} \\
& =\boldsymbol{A}(\boldsymbol{\theta}) \boldsymbol{h}_{B R}(\boldsymbol{\theta})+\hat{\boldsymbol{n}}
\end{aligned}
$$

$\boldsymbol{\Omega}$ is determined by the status of metamaterial elements. The proposed method can be realized even with 1-bit encoding RIS [19], the matrix elements in this case are only \pm 1 . Let $\Omega$ be the Hadamard matrix, then $\boldsymbol{\Omega}^{-1}=\frac{1}{M} \boldsymbol{\Omega}^{H}$.

We define the atomic set with the column vector of $\boldsymbol{A}(\boldsymbol{\theta})$

$$
\mathcal{A}=\left\{\boldsymbol{a}(\theta) \in \mathbb{C}^{N \times 1}: \theta \in\left[-\frac{\pi}{2}, \frac{\pi}{2}\right]\right\}
$$

The atomic norm of $\hat{\boldsymbol{h}}_{B A}$ is

$$
\left\|\hat{\boldsymbol{h}}_{B A}\right\|_{\mathcal{A}}=\inf \left\{\sum_{k}\left\|c_{k}\right\|_{2}: \hat{\boldsymbol{h}}_{B A}=\sum_{k} \boldsymbol{a}\left(\theta_{k}\right) c_{k}\right\}
$$

The ANM problem can be cast as the following semidefinite program (SDP) [20], [21]

$$
\begin{array}{lr}
\min _{\boldsymbol{X}, \boldsymbol{T}(\boldsymbol{u})} \operatorname{tr}(\boldsymbol{X})+\operatorname{tr}(\boldsymbol{T}) \\
\text { subject to } & {\left[\begin{array}{cc}
\boldsymbol{X} & \hat{\boldsymbol{h}}_{B A}^{H} \\
\hat{\boldsymbol{h}}_{B A} & \boldsymbol{T}(\boldsymbol{u})
\end{array}\right] \succeq \mathbf{0}}
\end{array}
$$

where $\boldsymbol{T}(\boldsymbol{u})$ is a Hermitian Toplitz matrix,

$$
\boldsymbol{T}(\boldsymbol{u})=\left[\begin{array}{cccc}
u_{1} & u_{2} & \cdots & u_{N} \\
u_{2}^{*} & u_{1} & \cdots & u_{N-1} \\
\vdots & \vdots & \ddots & \vdots \\
u_{N}^{*} & u_{N-1}^{*} & \cdots & u_{1}
\end{array}\right]
$$

$\boldsymbol{T}(\boldsymbol{u})$ can be decomposed into the following form,

$$
\begin{aligned}
\boldsymbol{T}(\boldsymbol{u}) & =\sum_{k=1}^{K} p_{k} \boldsymbol{a}\left(\theta_{k}\right) \boldsymbol{a}^{H}\left(\theta_{k}\right) \\
& =\boldsymbol{A}(\boldsymbol{\theta}) \boldsymbol{P} \boldsymbol{A}^{H}(\boldsymbol{\theta})
\end{aligned}
$$

the decomposition in (16) is unique if $K<N$. For RIS antenna, the condition can be satisfied as long as the number of elements is greater than the number of paths. So we can obtain the AoA of paths after solving SDP in (14) and decomposing $\boldsymbol{T}(\boldsymbol{u})$.

A subspace method such as ESPRIT or MUSIC can be used to compute the decomposition in (16). Taking the MUSIC method as an example, the eigenvalue decomposition is performed on $\boldsymbol{T}(\boldsymbol{u})$,

$$
\boldsymbol{T}(\boldsymbol{u})=\boldsymbol{U} \boldsymbol{\Lambda} \boldsymbol{U}^{H}
$$

where $\boldsymbol{U}=\left[\boldsymbol{u}_{1}, \ldots, \boldsymbol{u}_{L}, \boldsymbol{u}_{L+1}, \ldots, \boldsymbol{u}_{N}\right] . \quad \boldsymbol{G} \triangleq$ $\left[\boldsymbol{u}_{L+1}, \ldots, \boldsymbol{u}_{N}\right]$ are $N-L$ eigenvectors corresponding to the smaller eigenvalues, which compose the noise subspace. So it should be orthogonal to $\boldsymbol{A}(\boldsymbol{\theta})$,

$$
\boldsymbol{A}^{H}(\boldsymbol{\theta}) \boldsymbol{G}=\mathbf{0}
$$

We construct the peak search function

$$
f(\theta)=\frac{1}{\boldsymbol{a}^{H}(\theta) \boldsymbol{G} \boldsymbol{G}^{H} \boldsymbol{a}(\theta)}
$$

When there is a peak in the range of $\theta \in[-\pi / 2, \pi / 2]$, the corresponding position is estimated AoA $\tilde{\boldsymbol{\theta}}$. Then we substitute $\tilde{\boldsymbol{\theta}}$ into (11) to find the least square solution $h_{B R}(\tilde{\boldsymbol{\theta}})$, which is the estimated multipath channel gain.

\section{NumERICAL RESULTS}

In this section, we will demonstrate the performance of our scheme. If there is no special instruction, the parameters in the simulation are set as follows:

Alice and Bob communicate with each other, and both are equipped with a single antenna. Alice uses 1-bit coding RIS antenna. The metamaterial elements of the RIS antenna are arranged in a ULA with a spacing of 0.25 wavelength, and ehe number of elements is $N=32$. Bob uses an conventional omnidirectional antenna. In all simulations, the frame structure is shown in Figure 5. For the sake of simplicity and without loss of generality, let the channel remain unchanged within one frame period, the number of paths $L=3$, each path follows $\mathcal{C N}(0,1)$, and the sum of paths is also a complex Gaussian Distribution, namely Rayleigh fading channel. The angle spread of multipath is $\pi / 2$, centered on the normal direction of RIS antenna front, the minimum separation between paths is 5 degrees, and the AoAs follow a uniform distribution. Pilot length $K=16$, and we denote $P$ is the power of sending pilot signal $s_{k}$, the received signal-to-noise ratio (SNR) is

$$
\begin{aligned}
\mathrm{SNR} & =\frac{\operatorname{tr}\left(\mathbb{E}\left[\boldsymbol{A}(\boldsymbol{\theta}) \boldsymbol{h}_{B R}(\boldsymbol{\theta}) s_{k} s_{k}^{*} \boldsymbol{h}_{B R}^{H}(\boldsymbol{\theta}) \boldsymbol{A}^{H}(\boldsymbol{\theta})\right]\right)}{\operatorname{tr}\left(\mathbb{E}\left[\boldsymbol{n}_{k m} \boldsymbol{n}_{k m}^{H}\right]\right)} \\
& =\frac{\operatorname{tr}\left(\boldsymbol{A}(\boldsymbol{\theta}) \boldsymbol{h}_{B R}(\boldsymbol{\theta}) \boldsymbol{h}_{B R}^{H}(\boldsymbol{\theta}) \boldsymbol{A}^{H}(\boldsymbol{\theta})\right) P}{N}
\end{aligned}
$$

the SNR can be controlled by adjusting $P$, usually $5 \mathrm{~dB}$. The estimation performance is described by the normalized mean square error (NMSE), which indicates the deviation between estimated value and actual value. The NMSE of AoA and gain are defined as follows

$$
\begin{aligned}
& \operatorname{NMSE}(\boldsymbol{\theta})=\frac{\mathbb{E}\left[\|\boldsymbol{\theta}-\tilde{\boldsymbol{\theta}}\|_{2}^{2}\right]}{\mathbb{E}\left[\|\boldsymbol{\theta}\|_{2}^{2}\right]} \\
& \operatorname{NMSE}\left(\boldsymbol{h}_{B R}\right)=\frac{\mathbb{E}\left[\left\|\boldsymbol{h}_{B R}-\tilde{\boldsymbol{h}}_{B R}\right\|_{2}^{2}\right]}{\mathbb{E}\left[\left\|\boldsymbol{h}_{B R}\right\|_{2}^{2}\right]}
\end{aligned}
$$

where the elements of $\boldsymbol{\theta}, \tilde{\boldsymbol{\theta}}$ and $\boldsymbol{\theta}-\tilde{\boldsymbol{\theta}}$ are all in the range of $[-\pi, \pi] .10000$ Monte Carlo experiments are performed in each point to average the results.

\section{A. Effect of Training Overhead}

Figure 6 show that under different pilot lengths $K$, the estimation performance of the proposed method for AoA and path gain varies with the SNR and angle spread. Increasing the pilot length is equivalent to increasing the number of samples for averaging. Therefore, longer pilot sequence can bring better noise reduction effect, thereby improving the channel 
estimation performance, but the long pilot sequence will bring greater overhead. At low SNR, increasing pilot length can produce a relatively obvious performance improvement. But with the increase of SNR, the influence of pilot length on the NMSE will be reduced, almost no effect at high SNR.

When the angle spread is small, the probability of two paths reaching the receiver from a similar angle decreases as the increasing of angle spread. Therefore, the RIS antenna can distinguish multipaths more easily, and the estimation performance improves with the angular dispersion. Therefore, the RIS antenna can distinguish multipath more easily, thereby improving the estimation performance. But when the angle spread is large, especially close to $\pi$, there will be more cases where the angle between AoA and normal direction is larger, so the resolution decreases.

\section{B. Effect of the Number of Paths}

As shown in equation (4), the received signal can be regarded as the projection of paths. Therefore, whether the multipath can be separated or recovered is inevitable related to the number of paths $L$. Figure 7 show the variation of estimation performance with SNR and angle spread when the number of paths is different. The probability of collision between paths increases with $L$. When the difference of AoA is less than the minimum interval that can be accurately resolved, the resolution blur will occur [21], thereby reducing the estimation performance. When $L=1$, the estimated performance is less affected by the angular spread, but a larger incident angle will still cause resolution accuracy to decrease.

\section{Effect of the Number of Metamaterial Elements}

Increasing the number of metamaterial elements will increase the number of samples for electromagnetic wave in the spatial dimension, so it can improve the ability to resolve multipath angles for RIS antenna. Figure 8 show the variation of estimation performance with SNR and angle spread when the number of metamaterial elements is different. In this simulation, the size of entire RIS antenna is kept unchanged at 8 wavelength. The performance improved with the increase of $M$. However, since the aperture of RIS antenna remains unchanged, the increase of $M$ will result in a decrease of element spacing, thus limiting the performance improvement.

In addition, under the same SNR, the performance of AoA estimation is better than that of path gain. This is because the proposed scheme first estimates AoA, and then estimates path gain based on AoA, resulting in a certain accumulation of errors. How to design a better process to avoid error accumulation will be one of our future research focuses.

\section{Conclusion}

In this paper, we study the multipath separation and parameter estimation by single ris antenna in fading channel. For point-to-point single input single output (SISO) scenario, a multipath channel measurement method based on the agility of the RIS antenna pattern is proposed. By rapidly changing the 1-bit coding RIS antenna configuration parameters within one pilot symbol period to form an set of uncorrelated pattern, the fast sampling of the multipath channel is realized, and parameters are estimated from the sampled value by utilizing the spatial sparseness. Simulation results show that even if a single RIS antenna is used, the AoA and path gain can be estimated at the same time, and it has better spatial channel perception capabilities than conventional antennas. In addition, although we used one-dimensional ULA RIS antenna in this paper, it can be easily extended to two-dimensional planar arrangement, and this method is also suitable for reflecting RIS. The specific implementation of this aspect is for further studies.

\section{REFERENCES}

[1] M. Vanderveen, C. Papadias, and A. Paulraj, "Joint angle and delay estimation (JADE) for multipath signals arriving at an antenna array," IEEE Communications Letters, vol. 1, no. 1, pp. 12-14, Jan. 1997, conference Name: IEEE Communications Letters.

[2] Q. Wu and R. Zhang, "Towards Smart and Reconfigurable Environment: Intelligent Reflecting Surface Aided Wireless Network," IEEE Communications Magazine, vol. 58, no. 1, pp. 106-112, Jan. 2020.

[3] Z. Chen, X. Ma, C. Han, and Q. Wen, "Towards intelligent reflecting surface empowered 6G terahertz communications: A survey," China Communications, vol. 18, no. 5, pp. 93-119, May 2021, conference Name: China Communications.

[4] S. Hu, F. Rusek, and O. Edfors, "Beyond Massive MIMO: The Potential of Data Transmission With Large Intelligent Surfaces," IEEE Transactions on Signal Processing, vol. 66, no. 10, pp. 2746-2758, May 2018, conference Name: IEEE Transactions on Signal Processing.

[5] J. Chen, Y.-C. Liang, Y. Pei, and H. Guo, "Intelligent Reflecting Surface: A Programmable Wireless Environment for Physical Layer Security," IEEE Access, vol. 7, pp. 82 599-82 612, 2019, conference Name: IEEE Access.

[6] Z.-Q. He and X. Yuan, "Cascaded Channel Estimation for Large Intelligent Metasurface Assisted Massive MIMO,' IEEE Wireless Communications Letters, vol. 9, no. 2, pp. 210-214, Feb. 2020.

[7] B. Li, Z. Zhang, Z. Hu, and Y. Chen, "Joint Array Diagnosis and Channel Estimation for RIS-Aided mmWave MIMO System," IEEE Access, vol. 8, pp. 193 992-194006, 2020.

[8] L. You, J. Xiong, D. W. K. Ng, C. Yuen, W. Wang, and X. Gao, "Energy Efficiency and Spectral Efficiency Tradeoff in RIS-Aided Multiuser MIMO Uplink Transmission," IEEE Transactions on Signal Processing, vol. 69, pp. 1407-1421, 2021

[9] L. Zhang, M. Z. Chen, W. Tang, J. Y. Dai, L. Miao, X. Y. Zhou, S. Jin, Q. Cheng, and T. J. Cui, "A wireless communication scheme based on space- and frequency-division multiplexing using digital metasurfaces," Nature Electronics, vol. 4, no. 3, pp. 218-227, Mar. 2021.

[10] N. Shlezinger, G. C. Alexandropoulos, M. F. Imani, Y. C. Eldar, and D. R. Smith, "Dynamic Metasurface Antennas for 6G Extreme Massive MIMO Communications," IEEE Wireless Communications, pp. 1-8, 2021.

[11] N. Shlezinger, O. Dicker, Y. C. Eldar, I. Yoo, M. F. Imani, and D. R. Smith, "Dynamic Metasurface Antennas for Uplink Massive MIMO Systems," IEEE Transactions on Communications, vol. 67, no. 10, pp. 6829-6843, Oct. 2019, conference Name: IEEE Transactions on Communications.

[12] I. Yoo and D. R. Smith, "Dynamic Metasurface Antennas for HigherOrder MIMO Systems in Indoor Environments," IEEE Wireless Communications Letters, vol. 9, no. 7, pp. 1129-1132, Jul. 2020, conference Name: IEEE Wireless Communications Letters.

[13] L. Jin, Y. Lou, X. Xu, Z. Zhong, and H. Wang, "Separating Multi-Stream Signals Based on Space-Time Isomerism," in 2020 International Conference on Wireless Communications and Signal Processing (WCSP). Nanjing, China: IEEE, Oct. 2020, pp. 418-423.

[14] S. V. Hum and J. Perruisseau-Carrier, "Reconfigurable Reflectarrays and Array Lenses for Dynamic Antenna Beam Control: A Review," IEEE Transactions on Antennas and Propagation, vol. 62, no. 1, pp. 183 198, Jan. 2014, conference Name: IEEE Transactions on Antennas and Propagation. 

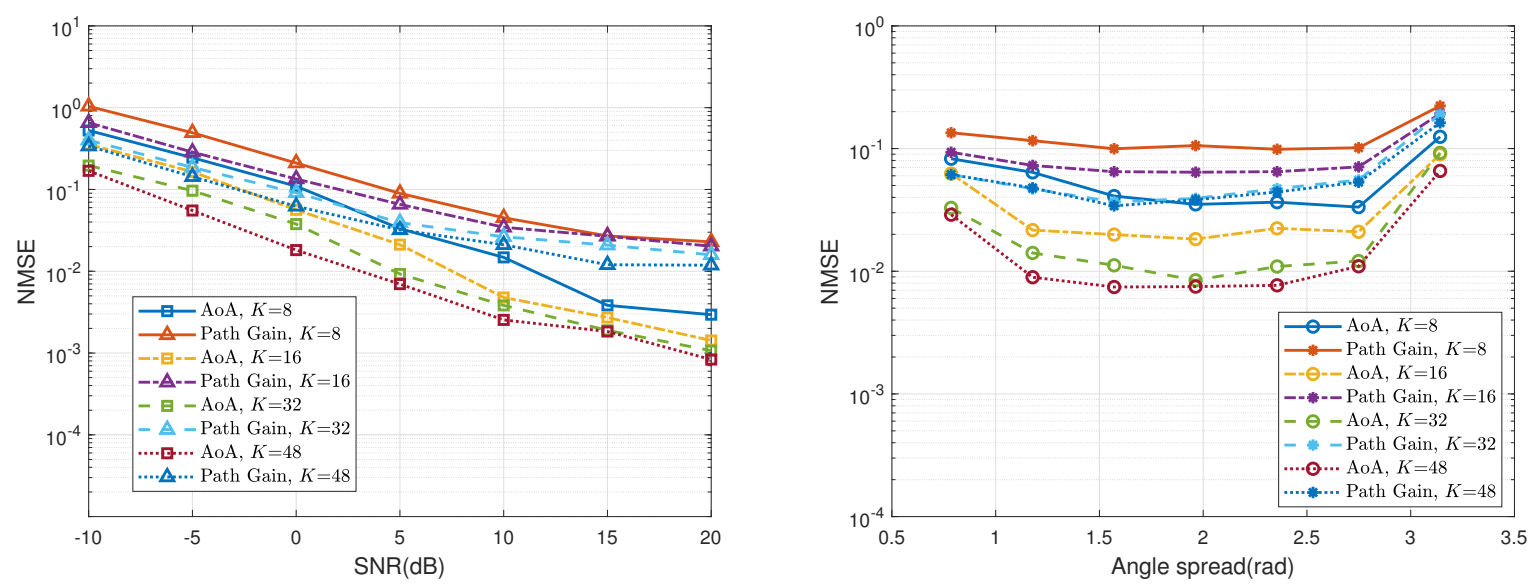

Fig. 6: Effect of pilot length on AoA and path gain estimation performance. Longer pilot sequence will reduce NMSE, but it will also bring greater overhead, resulting in lower communication rate.
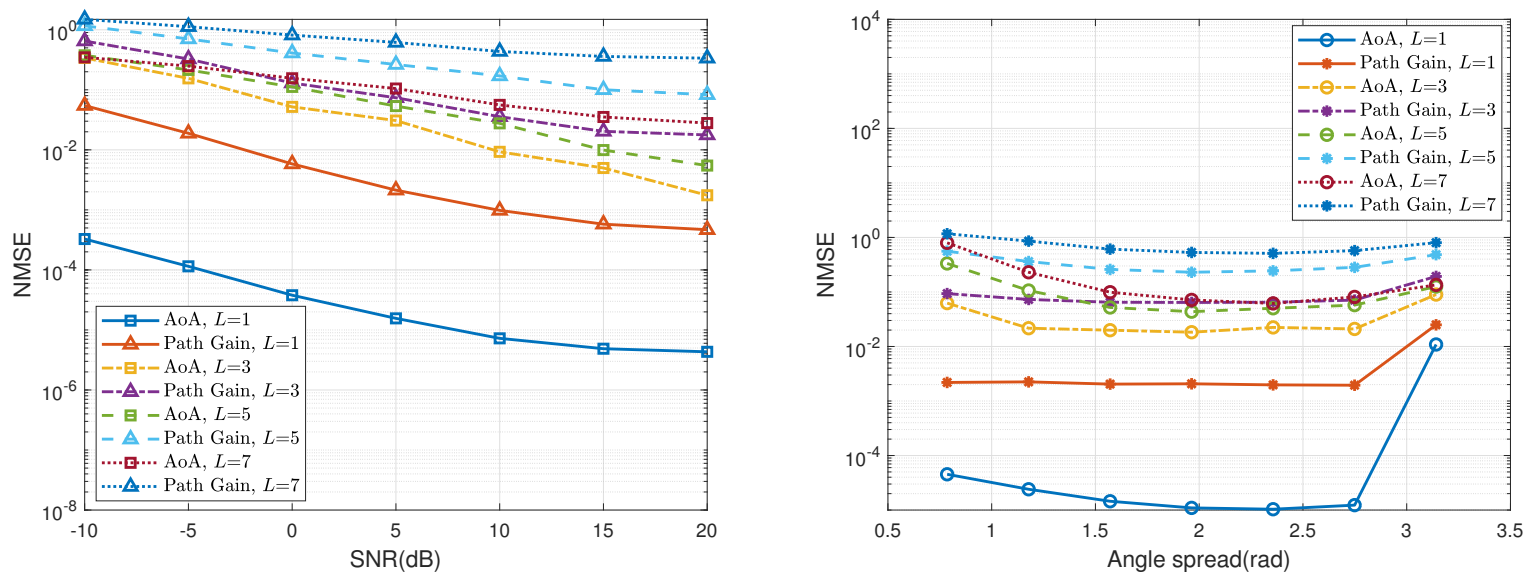

Fig. 7: Effect of the number of paths on AoA and path gain estimation performance. The probability of collision between paths increases with $L$, thereby reducing the estimation performance.
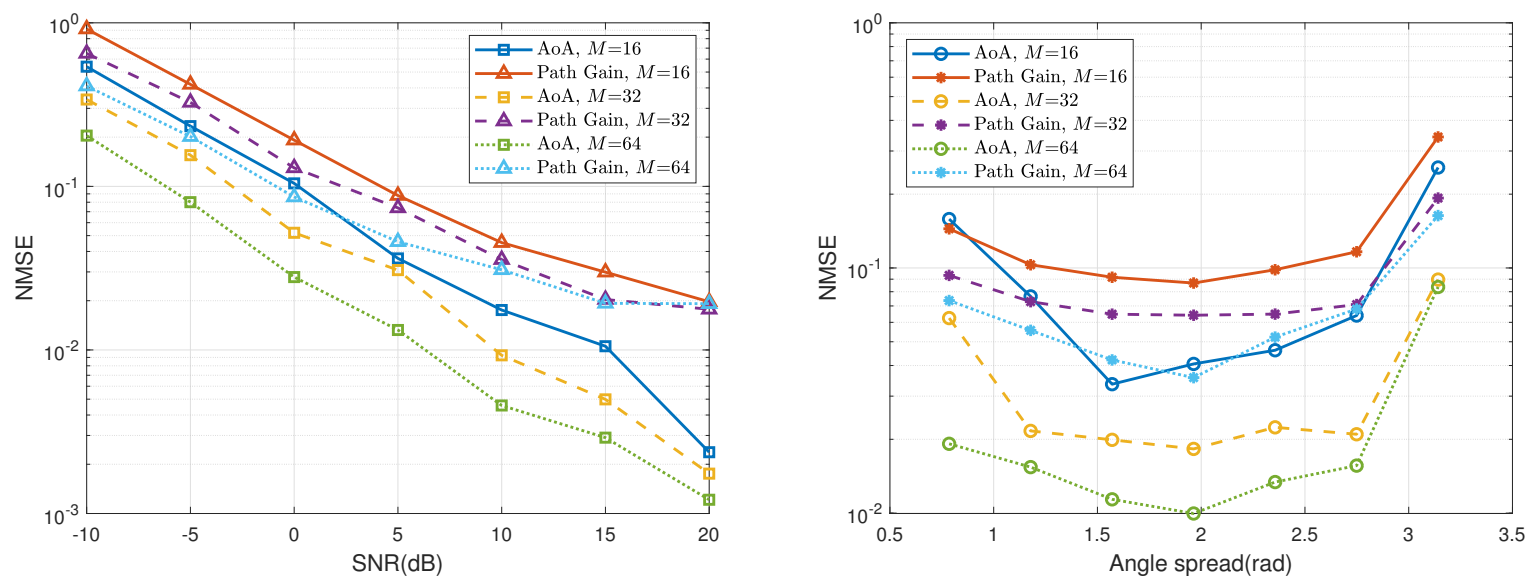

Fig. 8: Effect of the number of metamaterial elements on AoA and path gain estimation performance. The performance improved with the increase of $M$. But, the improvement will be limited when the aperture remains the same. 
[15] S. Fortunati, R. Grasso, F. Gini, M. S. Greco, and K. LePage, "Singlesnapshot DOA estimation by using Compressed Sensing," EURASIP Journal on Advances in Signal Processing, vol. 2014, no. 1, p. 120, Jul. 2014.

[16] P. Stoica, P. Babu, and J. Li, "New Method of Sparse Parameter Estimation in Separable Models and Its Use for Spectral Analysis of Irregularly Sampled Data," IEEE Transactions on Signal Processing, vol. 59, no. 1 , pp. 35-47, Jan. 2011, conference Name: IEEE Transactions on Signal Processing.

[17] — - "SPICE: A Sparse Covariance-Based Estimation Method for Array Processing," IEEE Transactions on Signal Processing, vol. 59, no. 2, pp 629-638, Feb. 2011, conference Name: IEEE Transactions on Signal Processing.

[18] Y. Zheng, L. Liu, and X. Yang, "SPICE-ML Algorithm for Directionof-Arrival Estimation," Sensors, vol. 20, no. 1, p. 119, Dec. 2019.

[19] R. Wu, L. Bao, L. Wu, and T. Cui, "Broadband transmission-type 1-bit coding metasurface for electromagnetic beam forming and scanning," Science China Physics, Mechanics \& Astronomy, vol. 63, no. 8, p. 284211, Aug. 2020

[20] Z. Yang and L. Xie, "Exact Joint Sparse Frequency Recovery via Optimization Methods," IEEE Transactions on Signal Processing, vol. 64, no. 19, pp. 5145-5157, Oct. 2016, conference Name: IEEE Transactions on Signal Processing.

[21] Z. Yang, "Direction-of-Arrival Estimation Using Atomic Norm Methods: Affects of Multiple Snapshots and Coherent Sources," in 2018 IEEE International Conference on Computational Electromagnetics (ICCEM). Chengdu, China: IEEE, Mar. 2018, pp. 1-3. 